\title{
Comparação entre qualidade de visão auto-relatada e acuidade visual em população idosa de baixa renda na cidade de São Paulo
}

\author{
Comparison between self-reported quality of vision and visual acuity in a \\ low-income elderly population in the city of São Paulo
}

\author{
Rafael Werneck Cinoto ${ }^{1}$ \\ Adriana Berezovsky ${ }^{2}$ \\ Rubens Belfort $\mathrm{Jr}^{3}{ }^{3}$ \\ Solang'e Rios Salomão ${ }^{4}$
}

\begin{tabular}{|l|}
\hline RESUMO \\
\hline Objetivo: Questionários de auto-avaliação visual vêm sendo utilizados em \\
pesquisas em vários campos da oftalmologia. O objetivo deste trabalho é \\
avaliar e correlacionar a qualidade visual auto-relatada por meio de ques- \\
tionário de função visual com a medida de acuidade visual. Métodos: 806 \\
indivíduos com idade igual ou acima de 60 anos, que faziam parte de \\
população avaliada em estudo epidemiológico de doenças oculares em \\
idosos foram entrevistados e submetidos à medida da acuidade visual para \\
longe e para perto e também foram submetidos a um questionário de função \\
visual. Resultados: Foram encontradas fracas correlações entre as acui- \\
dades visuais e as respostas do questionário. Conclusão: A qualidade \\
visual auto-relatada por meio de questionário de função visual não tem \\
correspondência direta com a medida da acuidade visual de um paciente, \\
mas pode trazer informações importantes para o oftalmologista.
\end{tabular}

Descritores: Visão/fisiologia; Acuidade visual; Erros de refração/diagnóstico; Autorevelação; Perfil de impacto da doença; Questionários
Trabalho realizado no Departamento de Oftalmologia da Universidade Federal de São Paulo - UNIFESP São Paulo (SP) - Brasil.

${ }^{1}$ Bacharel em Psicologia, Assistente de Pesquisa do Departamento de Oftalmologia da Universidade Federal de São Paulo - UNIFESP - São Paulo (SP) - Brasil. ${ }_{2}^{2}$ Professora Adjunto-Doutora do Departamento de Oftalmologia da UNIFESP - São Paulo (SP) - Brasil.

${ }_{3}^{3}$ Professor Titular do Departamento de Oftalmologia da UNIFESP - São Paulo (SP) - Brasil.

${ }^{4}$ Professora Associado Livre Docente do Departamento de Oftalmologia da UNIFESP - São Paulo (SP) - Brasil.

Endereço para correspondência: Solange Rios Salomão. Depto. de Oftalmologia da Universidade Federal de São Paulo - UNIFESP - Rua Botucatu, 822 - São Paulo (SP) CEP 04023-062

Recebido para publicação em 04.04.2005

Versão revisada recebida em 04.08.2005

Aprovação em 13.10.2005

Apoio financeiro: Secretaria de Ciência, Tecnologia, Turismo e Desenvolvimento Econômico do Estado de São Paulo, Convênio PROCESSO SCTDE-244/01.

\section{INTRODUÇÃO}

A acuidade visual é a medida da capacidade do indivíduo em reconhecer detalhes de um objeto no espaço. Por ser uma medida feita em condições clínicas controladas de distância, iluminação e contraste, a capacidade de reconhecimento de detalhes não testa adequadamente a visão em condições de iluminação indireta muito forte como em um dia ensolarado ou resultante da iluminação dos faróis de carros vindos em direção oposta ${ }^{(1)}$.

Muitas pessoas têm boa visão quando submetidas a testes clínicos, como acuidade visual para longe e para perto e sensibilidade ao contraste, mas quando uma auto-avaliação visual é utilizada, muitas relatam piores condições visuais do que as verificadas nos exames clínicos, mesmo sobre atividades importantes para elas no dia-a-dia, como dirigir ou ler. Se algumas perguntas forem feitas de rotina aos pacientes, as informações obtidas já podem alertar o oftalmologista para eventuais dificuldades com a visão que não podem ser constatadas nos exames oftalmológicos realizados no consultório ${ }^{(1-12)}$.

A oftalmologia utiliza rotineiramente medidas psicofísicas das funções visuais, sendo a mais comumente empregada a acuidade visual de optotipos, para representar as capacidades visuais do paciente. Muitos estudos clínicos de eficácia de terapêutica e estudos epidemiológicos dependem de 
medidas das funções visuais como variáveis primárias de estudo. Apesar das medidas das funções visuais serem necessárias na prática clínica oftalmológica, as mesmas não são mais consideradas suficientes, pois são limitadas por fatores como contraste máximo e não refletem adequadamente as condições reais de visão do dia-a-dia e o aspecto subjetivo da própria percepção do mundo pelo paciente ${ }^{(6)}$.

Desde 1980, vários questionários de auto-avaliação visual foram desenvolvidos ${ }^{(6)}$. O NEI-VFQ (Questionário de Função Visual do "National Eye Institute"), por exemplo, foi criado para tentar suprir essas limitações ${ }^{(2)}$, principalmente nos casos em que o paciente tem acuidade visual normal, mas mesmo assim está insatisfeito com seu bem estar por causa da visão ${ }^{(4)}$. A utilização de questionários vem sendo cada vez maior nas pesquisas oftalmológicas, mas ainda muito limitada em consultas médicas.

O conceito de "qualidade de vida" ainda é nebuloso, de difícil definição e mensuração ${ }^{(6)}$. A qualidade de vida dos pacientes tem diferentes fatores a serem interpretados e, uma vez que saúde é definida não apenas como ausência de doença, mas também como bem estar físico, mental e social, cada vez mais o fator "qualidade de vida" deve ser considerado ${ }^{(7)}$.

Buscando uma melhor compreensão da relação entre a subjetividade do estado visual auto-relatado e a acuidade visual, este estudo teve por objetivo analisar comparativamente as respostas obtidas de um questionário de função visual com a medida da acuidade visual para longe e perto.

\section{MÉTODOS}

\section{Participantes}

Participaram deste estudo 806 adultos com 60 anos ou mais de idade. O estudo foi aprovado pelo Comitê de Ética em Pesquisa da UNIFESP e seguiu os princípios da Declaração de Helsinque. Dos 806 participantes, 359 foram entrevistados nos dias 13 e 14/04/2002 e 447 foram entrevistados nos dias 13 e 14/07/2002 nas instalações do Centro de Referência do Idoso, órgão do governo do Estado de São Paulo, no bairro de São Miguel Paulista, no município de São Paulo. Dados demográficos como sexo, idade, renda mensal e escolaridade foram colhidos para cada participante.

\section{Procedimentos}

Após a coleta das informações de identificação do participante, foi aplicado o "Questionário simplificado sobre função visual" (Anexo 1). Este instrumento foi adotado neste estudo, por ser um questionário de fácil e rápida aplicação e ter sido anteriormente utilizado no Brasil ${ }^{(13)}$. Os entrevistadores foram orientados a ler as perguntas e em seguida cada alternativa para que o indivíduo pudesse escolher a alternativa que melhor expressasse sua condição, sem influência do entrevistador. A medida monocular da acuidade visual (AV) que se apresentava (com ou sem correção óptica conforme o que o

\section{Anexo}

1) Como está sua visão? (Com o uso de óculos e lentes quando for o caso) com os dois olhos abertos:
(1) Ótima ou boa
(3) Ruim
(2) Regular
(4) Péssima (cegueira)
(9) N.R.

2) $\mathrm{O}$ (a) $\mathrm{Sr}(\mathrm{a})$ acha que sua visão piorou há quanto tempo?
(1) Menos de 1 ano (exclusive)
(3) 3 a 5 anos
(2) 1 a 2 anos
(5) Não piorou
(9) Não sabe/não responde

3) $\mathrm{O}$ (a) $\mathrm{Sr}($ a) acha que sua visão, hoje dificulta suas atividades diárias?
(1) Nada
(3) Médio
(2) Pouco
(4) Muito
(9) N.R.

4) Como está sua visão para longe? Com óculos quando usar. O senhor(a) consegue:

(1) Ler letreiro de filme na TV

(2) Ver placa com nome da rua antes de atravessá-la

(3) Ver o letreiro com o nome ou $n^{\circ}$ do ônibus

(4) Reconhecer um amigo do outro lado da rua

(5) Ver o sinaleiro luminoso para atravessar a rua

(6) Ver à distância

(9) Não sabe/não responde

5) Como está sua visão para perto? Com óculos quando usar. O senhor(a) consegue:

(1) Ler as letras da bula de remédio ou enfiar a linha na agulha

(2) Ler jornal ou escolher arroz

(3) Ler livro ou revista

(4) Ver carta ou baralho

(5) Ler manchete de jornal

(6) Não sabe ler ou não lê

(7) Não vê de perto

(9) Não sabe/não reponde

6) Há quanto tempo fez o último exame dos olhos?
(1) Menos de 1 ano (exclusive)
(2) 1 a 2 anos
(3) 3 ou mais anos
(4) Nunca foi examinado
(9) Não sabe/não responde

7) Em que tipo de serviço você fez o último exame com médico oftalmologista?

(1) Particular

(2) INAMPS

(3) Centro de Saúde Estado ou Prefeitura

(4) IAMSP/Servidor Municipal

(5) Convênio

(6) $\mathrm{EPM} / \mathrm{HSP}$

(7) Outros

(9) Não sabe/não responde

8) Comprou os óculos receitados anteriormente?
(1) Sim, somente o de perto
(2) Sim, somente o de longe
(3) Sim, longe e perto
(4) Não, porque especifique

(5) Não foi receitado

(9) Não sabe/não responde

9) Melhorou a sua visão com os óculos novos?
(1) $\mathrm{Sim}$
(2) Não
(3) Não foi receitado
(4) Não comprou os óculos receitados
(9) Não sabe/não responde 
paciente utilizasse na época) foi feita na mesma visita. Foi utilizada a tabela impressa de optotipos de Snellen a 5 metros e a tabela de optotipos de Jaegger a $40 \mathrm{~cm}$ para perto. Os valores de acuidade visual foram transformados em logaritmo do mínimo ângulo de resolução (logMAR) para efeito de análise estatística dos dados.

Quatro (perguntas $\mathrm{n}^{\circ}$ 1, 3, 4 e 5) de um total de nove perguntas do questionário simplificado sobre função visual foram selecionadas por abordarem aspectos da visão de longe e de perto para uma comparação com as medidas de acuidade visual. Nas 1 e 3, a pessoa deveria escolher apenas uma das alternativas. Na pergunta 4 , o entrevistador lia as alternativas, que estão em ordem decrescente de dificuldade visual, uma por uma, da primeira até quando o paciente respondesse não. Então era assinalada a última alternativa para a qual a pessoa respondeu sim. Na pergunta 5, o método era o mesmo da pergunta 4, mas o entrevistador lia as alternativas, que estão em ordem decrescente de dificuldade visual até que o paciente respondesse sim. Então era assinalada a primeira alternativa para a qual a pessoa respondeu sim.

Os participantes foram alocados em quatro grupos de acordo com suas acuidade visuais com a melhor correção óptica, no melhor olho, medida para longe. Foram classificados como normais os que apresentaram acuidade visual igual ou melhor a 20/25 (0,1 LogMAR). Aqueles com acuidade visual entre 20/30 e 20/60 (0,18 e 0,52 LogMAR) foram classificados como de acuidade visual reduzida. Foram classificados como tendo visão baixa os que apresentaram acuidade visual entre 20/100 e 20/400 (0,7 e 1,3 LogMAR). E por fim, foram considerados cegos os pacientes que apresentaram acuidade visual inferior a 20/400 (1,3 LogMAR). A mesma alocação analogamente foi feita com a acuidade visual medida para perto.

\section{RESULTADOS}

Dos 806 entrevistados, 18 foram excluídos das análises por não possuírem um ou mais dados, sejam eles de acuidade visual, idade ou respostas dos questionários. A idade dos 788 participantes que tiveram seus dados analisados variou de 60 a 90 anos (média $=68,95$ anos $\pm 6,06$ anos), sendo que $586(74,4 \%)$ eram do sexo feminino e $202(25,6 \%)$ eram do sexo masculino. A renda mensal variou de 0 a 15 salários mínimos (média= 263,81 reais $\pm 267,61$ reais), sendo que $94 \%$ dos pacientes tinham renda menor do que 3 salários mínimos. E a escolaridade foi, no máximo, de curso primário completo para $92 \%$ dos pacientes.

Com relação à $\mathrm{AV}$ que se apresenta para longe, dos 788 participantes: $343(43,5 \%)$ apresentaram AV normal; 398 $(50,5 \%)$ apresentaram visão reduzida; $37(4,7 \%)$ apresentaram visão baixa e $10(1,3 \%)$ foram considerados cegos. A medida da AV para perto foi normal em 427 (54,2\%) dos participantes; $323(41,0 \%)$ apresentaram acuidade visual reduzida; $29(3,7 \%)$ apresentaram visão baixa e $9(1,1 \%)$ participantes foram considerados cegos para perto.

Em relação à pergunta $\mathrm{n}^{\circ} 1$ do questionário sobre função visual, apesar de 43,5\% dos atendidos apresentarem visão normal para longe, apenas $23,6 \%$ destes responderam que sua visão estava ótima/boa. Da mesma maneira, das pessoas que foram classificadas como cegas para longe, apenas 30\% classificaram sua visão como péssima (Tabela 1).

Da mesma maneira, ainda em relação à pergunta $\mathrm{n}^{\circ} 1$ do questionário sobre função visual, 54,2\% dos examinados apresentaram visão normal para perto, mas apenas $24,6 \%$ destes responderam que sua visão estava ótima/boa. Além disso, das pessoas que foram classificadas como cegas para perto, a maioria $(55,6 \%)$ classificou sua visão como apenas "ruim" ao invés de péssima (Tabela 2).

\begin{tabular}{|lrrrrr|}
\hline \multicolumn{6}{|l}{ Tabela 1. Respostas para a pergunta $\mathbf{n}-1$ do questionário sobre função visual, estratificadas pelos critérios de visão normal, reduzida, baixa } \\
ou cegueira para longe
\end{tabular}

\begin{tabular}{|c|c|c|c|c|c|}
\hline Ótima ou boa & 105 (24,6\%) & $45(13,9 \%)$ & $5(17,2 \%)$ & $0(0,0 \%)$ & 155 (19,7\%) \\
\hline Ruim & $111(26,0 \%)$ & $121(37,5 \%)$ & $9(31,0 \%)$ & $5(55,6 \%)$ & $246(31,2 \%)$ \\
\hline Péssima & 12 ( $2,8 \%)$ & $13(4,0 \%)$ & $2(6,9 \%)$ & $3(33,3 \%)$ & $30(3,8 \%)$ \\
\hline N.R. & $0(0,0 \%)$ & $3(0,9 \%)$ & $1(3,4 \%)$ & $0(0,0 \%)$ & $4(0,5 \%)$ \\
\hline
\end{tabular}


Para a pergunta $\mathrm{n}^{\circ} 3$ do questionário sobre função visual, que avalia como a visão dificulta as atividades diárias da pessoa, houve todo tipo de resposta, praticamente na mesma quantidade, dentro do grupo de pessoas com visão normal ou reduzida tanto para longe como para perto. As porcentagens variaram de $18,7 \%$ a $29,4 \%$ para cada resposta (nada, pouco, médio ou muito). Já entre as pessoas que foram classificadas com visão baixa, a maioria respondeu que a visão atrapalha muito suas atividades diárias, tanto para longe $(37,8 \%)$ como para perto $(48,3 \%)$. Observa-se que a maioria $(90 \%)$ das pessoas classificadas como cegas para longe disseram que a visão atrapalha muito suas atividades diárias, mas apenas $2 / 3$ das pessoas classificadas como cegas para perto disseram que a visão atrapalha muito suas atividades diárias (Tabelas 3 e 4).

Com relação à pergunta $\mathrm{n}^{\circ} 4$ do questionário sobre função visual, dos participantes que foram classificados com visão normal para longe, $57,7 \%$ disseram conseguir "ler letreiro de filme na TV", mas já não conseguem "ver placa com nome da rua antes de atravessá-la". E a porcentagem de frequiência das outras respostas foi diminuindo na ordem em que as respostas apareceram. Em relação às pessoas que foram classificadas com visão baixa para longe, as respostas que foram mais frequientes foram de pessoas que conseguiam ainda "ler letreiro de filme na TV" $(32,4 \%)$ e "ver o sinaleiro luminoso para atravessar a rua" $(24,3 \%)$. Dos que foram classificados como cegos para longe, metade não respondeu a questão 4 , mas dentre estes ainda houve alguns que responderam que podiam "ver placa com nome da rua antes de atravessá-la" e outros que responderam conseguir "ver o sinaleiro luminoso para atravessar a rua". Parece que a maioria dessas pessoas que responderam N.R. quis dizer que não enxergava à distância (Tabela 5).

Na pergunta $\mathrm{n}^{\circ} 5$ do questionário sobre função visual, dos participantes que foram classificados com visão normal para perto, $60,9 \%$ disseram conseguir "ler as letras da bula de remédio ou enfiar a linha na agulha", mas 5,6\% responderam que não vêem de perto. Entre as pessoas que foram classificadas com visão baixa para perto $27,6 \%$ disseram que conse-

\begin{tabular}{|lrrrr|}
\hline \multicolumn{7}{|l|}{ Tabela 3. Respostas para a terceira pergunta do questionário sobre função visual, estratificadas pelos critérios de visão normal, reduzida, } \\
baixa ou cegueira longe
\end{tabular}

\begin{tabular}{|c|c|c|c|c|c|}
\hline & Normal & Reduzida & Visão baixa & Cegueira & Total \\
\hline Nada & $118(27,6 \%)$ & $72(22,3 \%)$ & $7(24,1 \%)$ & $0(0,0 \%)$ & $197(25,0 \%)$ \\
\hline Médio & 89 (20,8\%) & $67(20,7 \%)$ & $4(13,8 \%)$ & $2(22,2 \%)$ & 162 (20,6\%) \\
\hline Muito & $103(24,1 \%)$ & $91(28,2 \%)$ & $14(48,3 \%)$ & $6(66,7 \%)$ & $214(27,2 \%)$ \\
\hline N.R. & $3(0,7 \%)$ & $3(0,9 \%)$ & $0(0,0 \%)$ & $0(0,0 \%)$ & $6(0,8 \%)$ \\
\hline
\end{tabular}

\begin{tabular}{|c|c|c|c|c|c|}
\hline Legenda TV & $198(57,7 \%)$ & $158(39,7 \%)$ & $12(32,4 \%)$ & $0(0,0 \%)$ & $368(46,7 \%)$ \\
\hline Nome do ônibus & $27(7,9 \%)$ & 37 ( 9,3\%) & $3(8,1 \%)$ & $0(0,0 \%)$ & $67(8,5 \%)$ \\
\hline Amigo do outro lado da rua & $16(4,7 \%)$ & $46(11,6 \%)$ & $5(13,5 \%)$ & $0(0,0 \%)$ & $67(8,5 \%)$ \\
\hline Farol & $11(3,2 \%)$ & $34(8,5 \%)$ & $9(24,3 \%)$ & $3(30,0 \%)$ & $57(7,2 \%)$ \\
\hline Não sabe/não responde & $13(3,8 \%)$ & $25(6,3 \%)$ & $3(8,1 \%)$ & $5(50,0 \%)$ & $46(5,8 \%)$ \\
\hline Total & $343(43,5 \%)$ & $398(50,5 \%)$ & 37 ( $4,7 \%)$ & $10(1,3 \%)$ & $788(100,0 \%)$ \\
\hline
\end{tabular}




\begin{tabular}{|lrrrr|}
\hline \multicolumn{2}{|l}{ Tabela 6. Respostas para a quinta pergunta do questionário sobre função visual, estratificadas pelos critérios de visão normal, reduzida, } \\
baixa ou cegueira para perto
\end{tabular}

guem "ler as letras da bula de remédio ou enfiar a linha na agulha", mas a mesma quantidade $(27,6 \%)$ também respondeu que não vê de perto. E por fim, daquelas classificadas como cegas para perto, a maioria disse que não vê como era de se esperar (Tabela 6).

O teste de correlação de Spearman mostrou pobre correlação da acuidade visual, tanto de perto como de longe, com as perguntas selecionadas e analisadas do questionário de função visual. Os coeficientes de correlação foram muito baixos e variaram de 0,10 a 0,24 (Tabela 7), com $\mathrm{p}<0,05$.

\section{DISCUSS ÃO}

O presente estudo mostra que exames clínicos oftalmológicos podem dar uma idéia incompleta do funcionamento visual do paciente, confirmando estudos anteriores. O acréscimo de algumas simples questões pode proporcionar um melhor entendimento dos sintomas do indivíduo. Isso porque os questionários podem nos dar informações que não podem ser percebidas pela acuidade visual ou por testes clínicos. Mudanças no ambiente (como aumento da iluminação ambiente, por exemplo) podem melhorar a visão, mesmo em pessoas mais velhas.

Observou-se que mesmo pessoas com acuidade visual melhor do que 20/25 (0,1 LogMAR) relatam ter dificuldades para realizar tarefas comuns como ler o letreiro de um filme ou ler a placa com o nome da rua antes de atravessá-la. Por outro lado, as pessoas que tinham acuidade normal para longe deveriam relatar que conseguem enxergar à distância enquanto que apenas a minoria $(3,5 \%)$ respondeu isso. A auto-avaliação da visão pode nos dizer muito mais sobre a qualidade de vida do paciente, do que a acuidade visual medida objetivamente.

Concordando com resultados anteriores, não houve forte

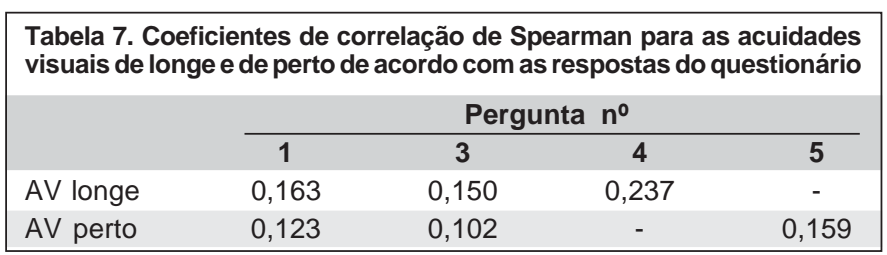

correlação da acuidade visual, nem de perto, nem de longe, com nenhuma pergunta analisada no questionário ${ }^{(6-7,10)}$. No entanto, vale ressaltar que este estudo analisou qualidade visual auto-relatada em pacientes idosos de baixa renda da cidade de São Paulo. A baixa escolaridade desta população pode ter sido um fator decisivo na escolha da melhor alternativa de resposta durante a administração do questionário.

Antes de se escolher um questionário deve-se considerar o propósito do mesmo. As perguntas selecionadas para análise advindas do questionário aplicado, podem ser muito importantes dependendo do tipo do estudo. Questionários de autoavaliação têm sido muito úteis se utilizados antes e depois de algum procedimento cirúrgico, por exemplo, para relacionar a satisfação da pessoa com a cirurgia ${ }^{(7,9)}$. Mas se feito isoladamente, não parece ter muito valor, pelo menos não com as alternativas possíveis que foram propostas nessa avaliação.

As diferenças encontradas nas respostas do questionário entre pessoas da mesma categoria de visão podem ser explicadas por alguns fatores. Por exemplo, todos podem ter algum grau de deficiência de acuidade visual, mas alguns também ter distúrbios cognitivos, outros ter limitações físicas, alguns ter distúrbios neurológicos e alguns ter problemas psiquiátricos. As respostas às perguntas podem depender mais desses problemas do que do impedimento visual propriamente dito. Glaucoma e retinopatia diabética, por exemplo, provavelmente têm outros efeitos, além da acuidade, que causam algum impacto na qualidade de vida ${ }^{(11)}$.

\section{CONCLUSÃO}

A qualidade de visão auto-relatada correlacionou-se pobremente com a medida da acuidade visual tanto para perto como para longe em população idosa de baixa renda da cidade de São Paulo. No entanto, questionários simples, com poucas perguntas, podem dar informações muito importantes para o oftalmologista numa anamnese em seu consultório ou numa pesquisa clínica. Como essas informações não podem ser obtidas através de exames clínicos, esses questionários deveriam ser indispensáveis como fonte complementar de informação da qualidade de visão do paciente. 


\section{ABSTRACT}

Purpose: Self-reported visual function questionnaires are becoming more common in clinical research. The purpose of this study is to evaluate and correlate self-reported visual function assessed by a visual function questionnaire with visual acuity. Methods: 806 patients aged 60 years and older, who were included in an epidemiological eye study, were interviewed and present visual acuity for near and distance was taken; they also answered a visual function questionnaire. Results: Weak correlations were found between self-reported visual function assessed by a visual function questionnaire and visual acuities for both distance and near. Conclusion: Self-reported visual function assessed by a visual function questionnaire did not correspond to visual acuity, nevertheless it can provide important information to the ophthalmologist.

Keywords: Vision/physiology; Visual acuity; Refractive errors/ diagnosis; Self disclosure; Aged; Sickness impact profile; Questionnaires

\section{REFERÊNCIAS}

1. Dick HB, Krummenauer F, Schwenn O, Krist R, Pfeiffer N. Objective and subjective evaluation of photic phenomena after monofocal and multifocal intraocular lens implantation. Ophthalmology. 1999;106(10):1878-86.

2. Berry S, Mangione CM, Lindblad AS, McDonnell PJ. Development of the
National Eye Institute refractive error correction quality of life questionnaire. Ophthalmology. 2003;110(12):2285-91.

3. Mangione CM, Berry S, Spritzer K, Janz NK, Klein R, Owsley C, Lee PP. Identifying the content area for the 51-item National Eye Institute visual function questionnaire: results from focus groups with visually impaired persons. Arch Ophthalmol. 1998;116(2):227-33.

4. Hays RD, Mangione CM, Ellwein L, Lindblad AS, Spritzer KL, McDonnell PJ. Psychometric properties of the National Eye Institute-refractive error quality of life instrument. Ophthalmology. 2003;110(12):2292-301.

5. Klein BEK, Klein R, Jensen SC. A short questionnaire on visual function of older adults to supplement ophthalmic examination. Am J Ophthalmol. 2000; $130(3): 350-2$

6. Massof RW, Rubin GS. Visual function assessment questionnaires. Surv Ophthalmol. 2001;45(6):531-48.

7. Atique D, Goulart DG, Lake JC, Lima FA, Felberg S, Nishiwaki-Dantas MC Qualidade de vida após transplante penetrante de córnea. Arq Bras Oftalmol. 2002;65(3):351-4

8. Nichols JJ, Mitchell GL, Saracino M, Zadnik K. Reliability and validity of refractive error-specific quality-of-life instruments. Arch Ophthalmol. 2003;121(9): 1289-96.

9. McDonnell PJ, Mangione C, Lee P, Lindblad AS, Spritzer KL, Berry S, et al. Responsiveness of the National Eye Institute refractive error quality of life instrument to surgical correction of refractive error. Ophthalmology. 2003; 110(12):2302-9.

10. Lamoureux EL, Hassel JB, Keeffe JE. The impact of diabetic retinopathy on participation in daily living. Arch Ophthalmol. 2004;122(1):84-8.

11. Broman AT, Munoz B, Rodriguez J, Sanchez R, Quigley HA, Klein R, et al. The impact of visual impairment and eye disease on vision-related quality of life in a Mexican-American population: proyecto VER. Invest Ophthalmol Vis Sci. 2002;43(11):3393-8.

12. Lee P, Smith JP, Kington R. The relationship of self-rated vision and hearing to functional status and well-being among seniors 70 years and older. Am J Ophthalmol. 1999;127(4):447-52.

13. Medina NH, Barros OM, Haro-Muñoz E, Magdaleno RL, Barros AJD, Ramos LR. Morbidade ocular em idosos da cidade de São Paulo - SP, Brasil. Arq Bras Oftalmol. 1993;56(5):276-83.

\section{$10^{\text {th }}$ International Strabismological Association Meeting - ISA/CLADE}

18 a 20 de Fevereiro de 2006

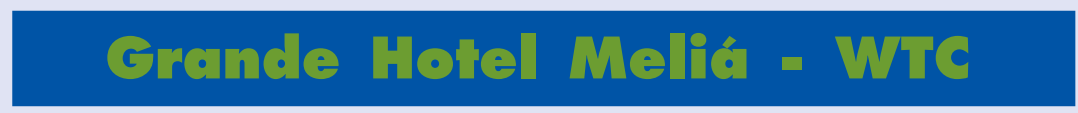

Sã॰ Paul• - SP

\section{INFORMAÇÕES: Home page: www.ophthalmology2006.com.br}

